http://jmscr.igmpublication.org/home/ ISSN (e)-2347-176x ISSN (p) 2455-0450

crossref DOI: https://dx.doi.org/10.18535/jmscr/v8i4.43

Journal Of Medical Science And Clinical Research

\title{
Adult umbilical hernia. Study of a series of 56 cases at the CHU in Libreville, Gabon
}

\author{
Authors \\ L S Bayonne Manou*, F K Diallo, K Dyatta Mayombo \\ Department of Visceral Surgery of the University Hospital Center of Libreville \\ *Corresponding Author \\ Doctor Bayonne Manou Louis Stanislas \\ BP: 5258 Libreville, Gabon
}

\begin{abstract}
Purpose: to report epidemiological, clinical and therapeutic results.

Material and Methods: It was a retrospective and descriptive study carried out in the visceral surgery department in 5 years (January 2015 to December 2019), on umbilical hernias in adults.

Results: Fifty-six patients underwent herniorraphy. The average age was 50 years old. The female gender was dominant (58.9\%). Physical work, multiparity, overweight were the predisposing factors. The aesthetics of the abdominal wall, mimic abdominal pain, hernial infatuation were the reasons for consultation. The dimensions of the collar were classified into 3 types: Small hernia: diameter of the collar $<2 \mathrm{~cm}(n=26 ; 46.5 \%)$; medium hernia: diameter between 2 and $4 \mathrm{~cm}(n=20 ; 35.7 \%)$; bulky hernia: diameter $>4 \mathrm{~cm}(n=10 ; 17.8 \%)$.

The approach was a laparotomy. The herniorraphy by simple suture $(n=22)$, the herniorraphy by plasty according to Mayo $(n=5)$ and prosthetic hernioplasty $(n=29)$ were the operating techniques. The polypropylene prosthesis was placed in 26 patients with retro aponeurotic pre-peritoneal. The results were excellent in 48 patients. The morbidity was 7 cases (12.5\%) including: 3 parietal infections, 2 seromas, 1 hematoma and 1 recurrence. There has been one death from a pulmonary embolism.

Conclusion: It is important to decide on a wall repair before complications arise. We support prosthetic hernioplasty in adult umbilical hernias.

Keywords: umbilical hernia, herniorraphy, hernioplasty, prosthesis.
\end{abstract}

\section{Introduction}

Umbilical hernia is common in our daily practice. The operative indication is often aesthetic, discomfort or local abdominal pain. The umbilical hernia presents as a reducible and painless navel mass. It may be barely visible or more obvious as a result of increased abdominal pressure ${ }^{[1]}$. The risk of strangulation makes hernia repair a common surgical practice in our department. It is a pathology most often benign but which can have complications involving life-threatening such as strangulation $^{[2]}$. In Libreville (Gabon), the umbilical hernia is quite common in the adult population, however it attracts little attention. The objective of this work is to report the epidemiological, clinical aspects, operating procedures and therapeutic results. 


\section{Patients and Methods}

It was a retrospective and descriptive monocentric study of simple or complicated umbilical hernias in adults. The variables studied were: epidemiological, clinical and therapeutic aspects. All of the information was gathered from hospital records and operative reports. The criteria for noninclusion were the other types of parietal hernias (white line, groin and Spiegel). All patients selected for implant placement received a single dose of $2 \mathrm{~g}$ of amoxicillin- clavulanic acid administered intravenously preoperatively. The data collected on pre-established cards were analyzed on an Excel table and by comparison of the percentages and the means.

\section{Results}

\section{Epidemiological aspects}

From January 2015 to December 2019, 56 consecutive primary umbilical hernias were collected in the General Surgery department in 5 years, an annual average of 11.2 cases. There were 33 women $(58.9 \%)$ and 23 men $(41.1 \%)$ with an average age of 50 years [33-71]. The sex ratio ( $\mathrm{F} /$ M) was 1.4. The medical history was dominated by multiparity in all women with an average parity of 2 , chronic constipation $(\mathrm{n}=19)$ and chronic cough $(n=7)$. On the professional level, we found the cultivators $(n=11)$, the workers $(n=12)$, the civil servants and the students $(n=7)$. Seventeen women and 5 men were overweight with a body mass index between 25 and 30 .

\section{Clinical and paraclinical aspects}

Thirty three patients consulted for aesthetic reasons, 18 for periodic local abdominal pain with discomfort and 5 cases for hernia infatuation. The patients were examined standing and supine. The dimensions (diameter) of the umbilical hernias were as follows: less than $1 \mathrm{~cm}(\mathrm{n}=26,46.5 \%)$; between $2-3 \mathrm{~cm}(\mathrm{n}=20,35.7 \%)$ and greater than $4 \mathrm{~cm}(\mathrm{n}=10,17.8 \%)$. The biological assessment and the radiography of the thorax were systematic and came within the framework of the anesthetic consultation. The radiography of the abdomen without preparation $(\mathrm{n}=3)$ and the abdominal CT $(n=2)$ were requested, in patients with a hernial symptomatology making suspect a craze.

\section{Therapeutic aspects}

All patients were operated under general anesthesia. The primary approaches were a left lateral midline laparotomy straddling the navel in $62.5 \%(\mathrm{n}=35)$ or a lower hemicircular cosmetic skin incision under the umbilical in $37.5 \%(\mathrm{n}=$ 21). This incision was more practiced in women for aesthetic reasons. The surgical techniques were: a simple herniorraphy $(\mathrm{n}=22)$, i.e. a resection of the sac associated with a simple closure of the umbilical ring, a herniorraphy with plasty performing a suture in overcoat according to the Mayo technique $(\mathrm{n}=5)$ or a prosthetic hernioplasty $(\mathrm{n}=29)$. The non-absorbable polypropylene prosthesis was placed openly in 26 patients in retro aponeurotic pre-peritoneal and 3 in pre-aponeurotic retro muscle in case of laborious dissection. The parietal repair was edgeto-edge suture autoplasty of the fascial banks. It was carried out by separate stitches with a nonabsorbable thread where a slow absorption. Five patients underwent emergency surgery for hernial infatuation, including two women in first trimester pregnancy. Postoperative oral prophylactic antibiotic therapy was initiated in all patients the day after the intervention. Preventive dose low molecular weight heparin thromboprophylaxis was recommended in at risk patients.

\section{Results of treatment}

The result was excellent in 48 patients. We found a morbidity rate of 7 cases (12.5\%): wall infection $(n=3)$, seroma $(n=2)$, hematoma $(n=1)$, recurrence $(\mathrm{n}=1)$. A death was caused by a pulmonary embolism in a diabetic. The length of hospital stay varied from one day to 5 days with an average stay of 2 days. Regular follow-up of the patients was 6 months [4 -13 months].

\section{Discussion}

\section{Epidemiological Aspects}

The umbilical hernia represents $6 \%$ of the entire abdominal wall in adults ${ }^{[3]}$. It is commonly diagnosed in general surgery, approximately $10 \%$ 
of all hernias of the abdominal wall are defined as an umbilical hernia and the prevalence in the adult population is $2 \%^{[4]}$. One study has shown that the umbilical hernia represents $16 \%$ of all hernias of the abdominal wall that have undergone surgical treatment ${ }^{[5]}$. The average age of our patients is 50 years with extremes ranging from 33 to 71 years. The female sex is predominant with a rate of $58.9 \%$. Our results can be superimposed on the literature ${ }^{[6,7]}$. Predisposing factors include extreme obesity, multiparity, and physical labor, cough and chronic constipation. These factors have already been reported by other authors ${ }^{[8]}$.

\section{Clinical and paraclinical aspects}

The abdominal aesthetic is the reason for the consultation of a large part of our cases. The rest of the patients had minimal intermittent abdominal pain that was seen by some as simple discomfort. The positive diagnosis of an umbilical hernia is clinical, the size of the collar varies from a simple centimeter orifice, giving rise to an intermittent swelling, to the voluminous hernia permanently externalized. From a surgical point of view, umbilical hernias can be classified into 3 types according to the diameter of the collar: Small hernia: diameter of the collar $<2 \mathrm{~cm}$; $\cdot$ Medium hernia: diameter between 2 and $4 \mathrm{~cm}$; $\cdot$ Bulky hernia: diameter $>4 \mathrm{~cm}^{[2]}$. The abdomen without preparation standing from the front and the abdominal computed tomography were requested in search of signs in favor of a complication such as hernial infatuation.

\section{Therapeutic aspects}

The choice of operative technique depended on the individual and the surgeon's preference. 51 patients $(91 \%)$ were operated on cold. These were planned interventions; the other 5 patients underwent emergency surgery for hernial infatuation. Median laparotomy straddling the navel was performed in 35 cases was dominant. We used 3 operating techniques: simple herniorraphy $(39,4 \%)$, herniorraphy according to Mayo's intervention (8,9\%), and hernioplasty with prosthetic reinforcement by conventional way (51.7\%). No laparoscopy has been done, we have no experience on this technique in our surgical team. It is generally accepted that repair by suture is sufficient for small hernias and that prosthetic repair for large hernias. The border between the two is not clearly identified; it is usually located 2 or $3 \mathrm{~cm} .{ }^{[2]}$. Simple herniorraphy requires resection of the bag associated with simple closure of the umbilical ring. Two objectives are to be sought: on the one hand to obtain a solid and functional repair of the abdominal wall, on the other hand the conservation of the umbilicus guarantees a good esthetic result.

The herniorraphy with plasty by the technique known as of "Mayo" realizes a suture in "overcoat" horizontally or vertically ${ }^{[9]}$.

Reinforcement of the wall with a non-absorbable prosthesis often seems necessary if one does not want to be exposed to the risk of recurrence, observed in 10 to $20 \%$ of cases after simple suture for this type of hernia ${ }^{[10,11]}$. When no plan of cleavage preperitoneal cannot be released, force is to place the prosthesis in retro muscular, in front of the posterior sheet of the sheath of the great rights. This technique was practiced in 3 cases during our study

\section{Results of Treatment}

The length of hospital stay varied from a single day to 5 days with an average stay of 2.5 days. Some teams perform surgery on small umbilical hernias on an outpatient basis and others may exceed the week of hospitalization when it comes to herniated pregnancies ${ }^{[12]}$. The operative suites were excellent in $85.7 \%$. Complications were dominated by parietal and seroma infection. The infection is favored by the occurrence of either a hematoma or necrosis of the skin, especially since it is a specific area (diabetes, obesity) ${ }^{[13]}$.

The seroma resolves over time and does not require any special treatment. Paul D. Colavita et al reported a rate of $3.6 \%$ in their study ${ }^{[14]}$.

Thromboembolic complications, although generally quite rare, they are among the most formidable since they are likely to jeopardize the life of the patient. One case was observed in our 
study resulting in death. Recurrence was noted in one case $(1.7 \%)$ after a Mayo-type plasty. Her diagnosis was clinical. Several factors have been implicated in the occurrence of recurrence: the size of the hernial opening, the surgical technique used, overweight, smoking, and many other comorbidities which have been confirmed by several studies in the literature ${ }^{[15,16]}$.

\section{Conclusion}

It is important to know how to decide on a surgical intervention before complications that increase morbidity and mortality occur. According to our study, we support herniorraphy with prosthetic reinforcement. The recidivism rate is low or even nonexistent. It deserves to be the gold standard in adult umbilical hernias.

\section{Conflict of interest}

The authors declare no conflict of interest.

Thanks.

The authors thank the patients who accepted the consultation of their medical files for carrying out this work.

\section{References}

1. Delamare J, Garnier D. Dictionnaire des termes de médecine, 29ème édition, juillet 2006 Edition Maloine- ISBN 222402956.

2. Pélissier E., Armstrong O., Ngo P.. EMC (Elsevier Masson SAS, Paris), Techniques chirurgicales- Appareil digestif,40-145, 2011.

3. Perrakis E, Velimezis G, Vezakis A et al . Une nouvelle technologie sans tension pour la réparation de la hernie ombilicale, en utilisant le Prolene Hernia Système -résultat précoce de 48 cas .Hernie 2003 ; 7 178-80.

4. Jairam A P, Kaufmann R, Muysoms F, Jeekel J, Lange J F. La faisabilité de l'anesthésie locale pour le traitement chirurgical de la hernie ombilicale : une revue systématique de la littérature .Hernie $2017 ; 21$ :223-31.

5. Chung Lucia P. S. Abdominal wall hernias: symptoms and outcome. MD thesis 2014. University of Glasgow.
6. Lau H, Patil N G. Hernie ombilicale chez l'adulte .Laparoscopique vs réparation ouverte. Surg Endos 2003 ; 17 : 2016-20.

7. Arroyo A., Garcia P, Pérez F., Andreu J. Randomized clinical trial comparing suture and mesh repair of umbilical hernia in adults. University hospital of Elche, Spain

8. Muschaweck U, Reparation de hernie ombilicale et épigastrique .Surg Clin North Am 2003 ;83 : 1207-21.

9. El Mourabit Hamza. La hernie ombilicale de l'adulte thèse $\mathrm{N}^{\circ} 015$. Royaume du Maroc. 2019.

10. Polat C, Dervisoglu A, Senyurek G et al. Umbilical hernia repair with prolene hernia system. Am J Surg 2005;190:61- 4.

11. Manaouil X. H, Regimbeau J.-M, Loriau J., Verhaeghe P. Les hernies ombilicales : traitement par la technique de la prothèse 'timbre-poste'. Annales de chirurgie 128 (2003) 563-66.

12. Halm J A. Experimental and Clinical Approaches to Hernia Treatment and Prevention. Thesis Erasmus Universiteit Rotterdam. 2007.

13. Delaunay F et al. Staphylococcus lugdunensis necrotizing fasciitis after abdominal dermolipectomy: report of two cases and review of the literature. Ann Chir Plast Esthet 2014 ; 59(2): 136-39.

14. Colavita P D., Belyansky I. Umbilical hernia repair with mesh: identifying effectors of ideal outcomes. Article. The american journal of surgery 2014.

15. Brancato G, Privitera A, Gandolfo L, Donati M, Caglia P. Plug technique for umbilical hernia repair in the adults. Minerva Chir 2002;57:13-6.

16. Sanajav P, Reid TD, Davies EL et al. Retrospective comparison of mesh and sutured repair for umbilical hernias. Hernia 2005;9:248 -51. 